•植物园定位与发展战略专题・

\title{
植物园与植物回归
}

\author{
任 海 $^{*}$ \\ (中国科学院华南植物园广东省应用植物学重点实验室, 广州 510650)
}

\begin{abstract}
摘要: 为了有效保护珍稀濒危植物, 植物园在迁地保护过程中开始关注迁地保护和野外回归相结合, 其拥有的活 植物资源、知识、技能和设施可为植物回归提供重要支撑。早期的植物回归实践注重用园艺手段增加种苗存活率, 后来强调了种群恢复, 并把植物回归放到生态系统恢复的背景下考虑, 近几年则强调了全球变化背景下的植物回 归。目前, 全世界植物园的回归研究和实践进展主要集中在影响回归植株定居的因素、回归的遗传多样性、全球 变化对回归的影响和成功回归的标准等四方面。在人类干扰和全球变化情景下, 植物园需要综合考虑就地保护、 迁地保护和回归三位一体的综合保育体系, 以实现植物多样性的有效保护。
\end{abstract}

关键词: 植物回归; 植物园; 珍稀濒危植物; 全球变化

\section{The role of botanical gardens in reintroduction of plants}

\author{
Hai Ren ${ }^{*}$ \\ Guangdong Provincial Key Laboratory of Applied Botany, South China Botanical Garden, Chinese Academy of Sciences, \\ Guangzhou 510650
}

\begin{abstract}
In order to effectively protect rare and endangered plants, botanical gardens have moved from $e x$ situ conservation to the combination of ex situ conservation and reintroduction. Its living collections, knowledge, skills, and facilities can be used in the process of plant reintroduction. Early plant reintroduction practices have focused on increasing the survival rate of seedlings or seeds by means of horticulture, and later emphasized population recovery and plant reintroduction in the context of ecosystem restoration. In recent years, emphasis has been placed on plant reintroduction in the context of global change. Nowadays, plant reintroduction research and practice at botanical gardens is mainly focused on factors affecting reintroduced individuals, genetic diversity associated with reintroduction, the impacts of global change on reintroduction, and the criteria for successful reintroduction. With human disturbance and global change, botanical gardens need to take into account integrating in situ conservation, ex situ conservation, and reintroduction to effectively protect plant diversity.
\end{abstract}

Key words: plant reintroduction; botanical gardens; rare and endangered plants; global change

\section{1 植物园与珍稀濒危植物保护}

植物园 (botanical/botanic garden)意指“植物学 的园地”。世界上第一个植物园是1545年在意大利 建立的Padua大学药用植物园。国际植物园协会 (International Association of Botanic Gardens, IABG) 1963年将植物园定义为: 一个向公众开放的、其内 的植物标有铭牌的园地。国际植物园保护联盟
(Botanic Gardens Conservation International, BGCI) 将植物园分为 12 种类型, 其中, 经典类型的植物园 是指立足于活植物的规范化收集和管理, 并基于这 些植物开展科学研究、资源保护、园林展示和公众 教育的机构(贺善安等, 2005; Wyse Jackson \& Sutherland, 2013)。现代意义上的植物园起源于栽培、研 究和试验药用植物, 历经了药用植物园、热带植物 园、欧洲经典植物园、市政植物园和特殊类型植物

收稿日期: 2017-06-16; 接受日期: 2017-09-17

基金项目: 国家重点研发计划项目(2016YFC1403002)和中国科学院 A 类战略性先导科技专项(XDA13020500)

* 通讯作者 Author for correspondence. E-mail: renhai@scib.ac.cn 
园(包括农业植物园、园艺植物园、种质资源收集园 等)等发展阶段。目前, 全球有3,300多个植物园, 中 国则有195个(任海和段子渊, 2017)。发达国家的植 物园在加强研究的基础上, 更倾向于综合保护生物 多样性和环境教育, 而发展中国家的植物园研究力 量还不够强, 在进行珍稀濒危植物综合保护的同时, 加强了经济植物的利用, 也有一定的科普能力(任 海, 2006; 任海和段子渊, 2017)。

植物园以植物的引种驯化工作为主, 收集了众 多的植物资源, 通过科学的造园方法, 展示优美的 园林景观。因此, 植物园不仅成为植物多样性研究、 保护、科普与利用的重要基地, 更是开展包括植物 繁殖、野外回归(reintroduction, 也译为再引种)、退 化生境的恢复等内容的物种恢复计划的重要场所 (Akeroyd \& Wyse Jackson, 1995)。《植物园保护国 际议程》提出植物园的使命是: (1)使公众意识到植 物多样性价值及其所面临的威胁; (2)采取实际行动 改善全球自然环境; (3)为了我们的子孙后代, 促进 和保证全球自然资源的可持续利用, 遏制植物种类 和其遗传多样性丧失并阻止全球自然环境的进一 步退化(许再富, 2005; Wyse Jackson \& Sutherland, 2012)。

最近几十年来, 由于人类活动和气候变化导致 生物多样性大量丧失, 植物园因为有活植物收集及 其保护设施而成为本土植物和珍稀濒危植物的迁 地保育中心(Heywood, 2011)。BGCI调查发现, 大部 分野生植物在《生物多样性公约》(CBD) 签署之前 就已在植物园栽培, 许多植物园也将珍稀濒危植物 保护作为重要的责任, 建立了珍稀濒危植物的专类 收集区并有可能开展野外回归(Wyse Jackson \& Kennedy, 2009)。中国目前的珍稀濒危植物包括: 国 家重点保护野生植物名录(农业部1999年颁布, 第 一批, 包括246种及 8 个类群)、中国生物多样性红色 名录一高等植物卷(环境保护部和中国科学院联合 发布, 采用2001年IUCN红色名录等级和标准, 对 34,450 种高等植物进行了评估, 有约 $11 \%$ 即3,767种 受到不同程度的威胁)、极小种群野生植物(国家林 业局2012年发布)等3大类。这些珍稀濒危植物大多 为我国特有, 具有不可替代的生态、经济、科学和 文化价值，急需实施这些物种的恢复计划。

BGCI数据表明全球 30 多万种高等植物中有 10 万多个栽培在植物园, 其中珍稀濒危植物 1 万多种
(Sharrock, 2012)。中国的植物园和树木园引种保存 了中国植物区系中约22,000种植物, 其中第一批国 家重点保护植物已有85\% (约270种)被引种保存(黄 宏文和张征, 2012)。由BGCI等制订的《全球植物保 护战略2011-2020》(Global Strategy for Plant Conservation 2011-2020, GSPC, www.plants2020.net)提 出了 16 个目标, 其中目标8为: 到2020年, 全球至少 $75 \%$ 的受威胁植物得到迁地保护, 最好是在原产地, 并将其中至少 $20 \%$ 列入恢复方案(回归)。为了有效 保护珍稀濒危植物, 植物园已从收集评估、迁地保 护转向就地保护、迁地保护和野外回归并重的综合 性物种保护上来。

\section{2 植物回归}

对珍稀濒危和乡土植物的保护主要有就地保 护、近地保护、迁地保护和植物回归。植物回归是 野生植物种群重建的重要途径, 是迁地保护与就地 保护的桥梁, 其保护效果超出了单纯的就地保护和 单一的物种保护。国际上一般把濒危植物和具有重 要经济、文化或生态价值的种类列为优先回归类 群。至2012年, 欧美国家约开展了700个分类群的回 归实践, 其中的 301 个植物种类中有 128 个获得了成 功(Ren et al, 2014); 中国开展了154种植物的回归, 大部分取得了成功(Liu et al, 2015)。

把植物从栽培环境移入经过改造的半自然或 野生环境中已有很长的历史, 林业上把树苗种在现 存植被中也有几百年的历史。现代意义上的回归研 究和实践起源于 20 世纪 60 年代的动物回归野外工 作, 植物的回归工作则是在 20 世纪70年代末 80 年代 初生物多样性保护受到重视之后, 才由植物园做起 来的。目前已知最早报道的植物回归案例于1979年 发生在西班牙(Sainz-Ollero \& Hernandez-Bermejo, 1979; Maunder, 1992)。由于植物园是在调查评价植 物野外生存状况及保护效果的基础上开展迁地保 护工作, 在迁地保护成功后, 欧美的植物园在 $20 世$ 纪80年代初就开始把迁地保护过程中生产的苗木 或种子回归到野外, 用于就地保护实践。早期的回 归注重用园艺手段增加苗木存活率, 后来强调了种 群恢复, 再后来把回归放到生态系统尺度上考虑, 近几年强调了全球变化背景下回归的研究。欧美的 植物园就在回归实践中形成了一定的程序(Falk et al, 1996)。欧洲理事会(1985)、BGCI (Akeroyd \& 
Wyse Jackson, 1995)、IUCN (1998, 2009), Maschinski 和Haskins (2012)先后出版了回归的程序或指南, 其 主要内容包括: 回归的目标、如何选择回归的物种、 回归的生境要求、回归的植物材料要求、植物种群 的调控、回归后的管理与监测、回归成功的标准、 回归的步骤等。

植物的回归是在迁地保护的基础上，通过人工 繁殖把植物引入到其原来分布的自然或半自然的 生境中, 以建立具有足够的遗传资源来适应进化改 变、可自然维持和更新的新种群。回归有如下4种 类型：(1)增强回归(augmentation, reinforcement或 enhancement), 即在现有野生种群内引入同一物种 的个体, 以增加该种群的生存能力。(2)回归 (reintroduction), 即狭义的“回归引种”, 在拟回归物 种的历史分布区范围内, 再引入该物种新的个体, 重建已消失的种群。(3) 异地回归(translocation, conservation introduction), 指在历史分布范围以外 相似生境中开展的回归。(4)重建回归(restitution, reestablishment或restoration), 指通过人工修复那些 受到破坏的种群, 使其尽可能恢复到历史的状态。 前两种回归集中在物种尺度, 而且是在已知范围内 进行的种群恢复; 而后两种是生态系统尺度的工作 (Ren et al, 2014)。

\section{3 植物园的植物回归实践与研究}

从世界范围看, 植物回归的研究与实践主要发 生在植物园, 这主要是因为植物园可为植物回归提 供全方位的知识、植物材料、技术支撑与人力资源, 例如: 植物园的引种信息系统、标本馆、种子库、 组织培养室等设备可为回归提供信息和材料准备; 植物园的各类保护工作和活植物收集更是回归的 基础, 且目前植物园承担的基于活植物收集的科研 项目多与迁地保护和回归有关; 植物园的园艺技能 可以提高回归植物的存活率; 植物园的科学研究有 植物分类学、植物形态学、植物解剖学、植物细胞 学、植物遗传学、植物生理学、物候学、园林园艺 学、全球变化、恢复生态学、生物化学、个体生态 学和繁殖生物学等, 这些知识是指导回归时所需要 的生态生物学来源, 更重要的是从事这些工作的科 技人员可以承担科学的回归工作(Raven, 2006; 任 海, 2006); 植物园的环境教育和科普活动还可为回 归提供公众参与机会或争取社会资金的支持。当前
发达国家植物园在国际植物园保护联盟的倡导下， 在履行《生物多样性公约》过程中, 实施了《全球 植物保护战略》, 考虑了全球变化、社区可持续发 展的影响, 更加关注了物种尺度甚至是生态系统尺 度的保护和恢复, 综合考虑了就地保护、迁地保护、 野外回归、资源利用等手段, 植物园在这个综合保育 体系中起了主导作用。

目前全世界植物园的回归研究进展主要集中 在影响回归植株定居的因素、回归的遗传多样性、 全球变化对回归的影响和成功回归的标准等4方 面。研究发现, 繁殖体类型、材料来源、种源是直 接野外收集还是贮藏、种源的数量及处理、不同种 群与地点的适合度、回归地点、生境观测数据、生 境的处理、种植时间等都对回归植物的成功定居有 影响(Guerrant \& Kaye, 2007)。回归时会因居群的时 空隔离而导致高的遗传分化和减少遗传多样性丧 失风险, 但增强型回归会导致亚种群间遗传同质化 并降低遗传多样性(Ren et al, 2010; Wang et al, 2013)。在全球变化情况下, 回归可以增加一个物种 的分布和多度, 改进基因流, 加强复合种群动态并 降低种群灭绝的风险(Falk et al, 1996)。回归种群也 面临着外来种入侵、生境破碎化、气候变化等影响。 为了减少这些影响, 拟回归物种的历史分布区不再 是回归时的唯一考虑地点, 可利用生境分布模型为 拟回归的物种找到合适的地点开展异地回归，这类 模型会考虑回归过程中的生态相似性、源种群的遗 传性以及生境质量的影响3个重要因素(Ren et al, 2014)。关于成功回归的标准虽然还未形成共识, 但 一般会分为短期和长期成功, 短期成功是指回归个 体的成活、种群的建立和扩散; 而长期成功是指回 归种群能自我维持并在生态系统中发挥功能作用。 最成功的回归包括产生二代个体, 回归种群融入种 群动态、种群遗传、个体行为、生态系统功能等生 态系统过程(Andel \& Aronson, 2012)。

在中国, 虽然林业系统在中国珍稀濒危植物的 保护中发挥着管理作用, 但中国的植物园却是植物 回归研究和实践的主要单位(表1), 比如: 中国科学 院武汉植物园在三峡工程前后, 系统开展了库区受 影响的疏花水柏枝(Myricaria laxiflora)等82种珍稀 植物的异地回归; 深圳仙湖植物园结合扶贫工作在 广西成功开展了德保苏铁(Cycas debaoensis)的回归; 中国科学院华南植物园系统开展了报春苣苔 
表1 中国植物园开展的植物回归案例

Table 1 The plant reintroduction cases from Chinese botanical gardens

\begin{tabular}{|c|c|}
\hline 植物种类 Species & 单位 Institution \\
\hline 仙湖苏铁 Cycas fairylakea & 中国科学院华南植物园 South China Botanical Garden, Chinese Academy of Sciences \\
\hline 水松 Glyptostrobus pensilis & 中国科学院华南植物园 South China Botanical Garden, Chinese Academy of Sciences \\
\hline 观光木 Tsoongiodendron odorum & 中国科学院华南植物园 South China Botanical Garden, Chinese Academy of Sciences \\
\hline 猪血木 Euryodendron excelsum & $\begin{array}{l}\text { 中国科学院华南植物园和云南大学 South China Botanical Garden, Chinese Academy of } \\
\text { Sciences and Yunnan University }\end{array}$ \\
\hline 丹霞梧桐 Firmiana danxiaensis & 中国科学院华南植物园 South China Botanical Garden, Chinese Academy of Sciences \\
\hline 四药门花 Tetrathyrium subcordatum & 中国科学院华南植物园 South China Botanical Garden, Chinese Academy of Sciences \\
\hline 报春苣苔 Primulina tabacum & 中国科学院华南植物园 South China Botanical Garden, Chinese Academy of Sciences \\
\hline 长梗木莲 Manglietia longipedunculata & 中国科学院华南植物园 South China Botanical Garden, Chinese Academy of Sciences \\
\hline 虎颜花 Tigridiopalma magnifica & 中国科学院华南植物园 South China Botanical Garden, Chinese Academy of Sciences \\
\hline 伯乐树 Bretscheneidera sinensis & 中国科学院华南植物园 South China Botanical Garden, Chinese Academy of Sciences \\
\hline 杜鹃红山茶 Camellia azalea & 中国科学院华南植物园 South China Botanical Garden, Chinese Academy of Sciences \\
\hline 德保苏铁 Cycas debaoensis & $\begin{array}{l}\text { 深圳市中国科学院仙湖植物园 Fairylake Botanical Garden, Shenzhen \& Chinese Academy } \\
\text { of Sciences }\end{array}$ \\
\hline 景宁木兰 Magnolia sinostellata & $\begin{array}{l}\text { 深圳市中国科学院仙湖植物园 Fairylake Botanical Garden, Shenzhen \& Chinese Academy } \\
\text { of Sciences }\end{array}$ \\
\hline 疏花水柏枝 Myricaria laxiflora & 中国科学院武汉植物园 Wuhan Botanical Garden, Chinese Academy of Sciences \\
\hline 荷叶铁线蕨 Adiantum nelumboides & 中国科学院武汉植物园 Wuhan Botanical Garden, Chinese Academy of Sciences \\
\hline 麻栗坡兜兰 Paphiopedilum malipoense & 中国科学院昆明植物园 Kunming Botanical Garden, Chinese Academy of Sciences \\
\hline 三棱栋 Trigonobalanus doichangensis & 中国科学院昆明植物园 Kunming Botanical Garden, Chinese Academy of Sciences \\
\hline 漾濞械 Acer yangbiense & 中国科学院昆明植物园 Kunming Botanical Garden, Chinese Academy of Sciences \\
\hline 白旗兒兰 Paphiopedilum spicerianum & $\begin{array}{l}\text { 中国科学院西双版纳热带植物园 Xishuangbanna Tropical Botanical Garden, Chinese } \\
\text { Academy of Sciences }\end{array}$ \\
\hline 凤蝶兰 Papilionanthe teres & $\begin{array}{l}\text { 中国科学院西双版纳热带植物园 Xishuangbanna Tropical Botanical Garden, Chinese } \\
\text { Academy of Sciences }\end{array}$ \\
\hline
\end{tabular}

(Primulina tabacum) 等28种华南珍稀濒危植物的回 归并总结出了一套回归模式, 发表了大量论文, 昆 明植物园成功开展了麻栗坡䚁兰 (Paphiopedilum malipoense)等6种极小种群野生植物的回归(孙卫邦, 2013)。此外, 中国还建立了 “选取适当的珍稀植物, 进行基础研究和繁殖技术攻关, 再进行野外回归和 市场化生产, 实现其有效保护, 加强公众的保护意 识, 同时通过区域生态规划及国家战略咨询, 推动 整个国家珍稀濒危植物回归工作” 的模式; 这种模 式初步实现珍稀濒危植物产业化, 产生了良好的社 会、生态和经济效益(Ren et al, 2012)。

发达国家成功回归的 128 个植物类群中, 约 $20 \%$ 是重建回归, $30 \%$ 是增强回归。美国植物保护中 心国际植物回归登录系统中有较全记录的 49 个案 例中, 92\%成活, 76\%达到繁殖状态, 33\%已产生后 代, 16\%的下一代又产生了下一代; 这些种中, 美国 栗子(Castanea dentata)回归的工作因做得最系统而
成了经典回归案例(Jacobs et al, 2013); 夏威夷在外 来种严重入侵后的生态恢复中实施了许多乡土种 的回归工作(Falk et al, 1996)。欧洲开展了234种植物 的回归工作, 但成功的不多(Godefroid et al, 2011)。 澳大利亚完成了 54 个种的回归工作, 只有 23 个成功, 动物的影响是导致失败的主因(Sheean et al, 2012)。 新加坡植物园利用组培技术在乡土兰花的回归方 面取得巨大的成功(Yam et al, 2010)。

\section{4 植物园的回归工作展望}

自20世纪80年代以来, 回归研究与实践工作日 益增多, 近些年已呈指数性增长, 因此, 有学者提 出了回归生物学(Reintroduction Biology)这个学科 (Maschinski \& Haskins, 2012)。可以预期, 回归生物 学的提出会促进相关工作。当前, 植物回归的研究 与实践有如下特点或趋势。

植物成功回归的标准是至少要产生下一代的 
植株, 这是一个长期的过程。但目前许多回归案例 缺乏长期监测数据, 失败的案例又无报道, 再加上 研究者间的数据共享少(Godefroid \& Vanderborght, 2011), 因此, 可能放大了 “短期成功”的效应, 也不 利于回归工作的理论总结。此外, 回归工作还主要 集中在实践方面, 理论研究还不够深入, 大部分研 究还是验证植物生态学、保育遗传学等学科的理论, 这些研究也主要涉及少数生态因子, 缺乏多生态因 子实验和复杂成分的理论分析。未来一段时间内, 植物园在开展回归工作时, 除了实施更多更系统的 案例外, 加强长期监测和信息共享是非常必要的, 当然也要进行理论总结。

Armstrong和Seddon (2008)认为物种回归研究 还有如下 10 个关键科学问题未解决: (1)回归群体的 大小和组成如何影响种群的建立? (2)回归前后的人 工管理如何影响种群的存活和扩散? (3)回归种群可 持续发展需要什么样的生境条件? (4)遗传多样性如 何影响回归种群的可持续性? (5)采集回归材料时对 原种群造成多大影响合适? (6)异地回归时如何选择 最佳地点? (7)异地回归可否用于种群隔离的补偿? (8)目标种和伴生种是否是这个生态系统中的乡土 种? (9)回归种及伴生种如何影响生态系统? (10)回 归种如何最终影响生态系统中的物种组成? 目前, 这些问题仍然未完全解决, 植物园可以利用自身的 优势, 开展系统的研究。

珍稀濒危植物的回归是种群尺度的工作, 还需 要放在群落、生态系统甚至景观尺度上考虑种群的 恢复。生态系统的结构与功能、地上与地下关系、 生物种间关系或食物网的恢复会有助于回归种群 的可持续存活。目前回归主要集中在物种个体的存 活和生境的营造上, 较少关注种群的建立和动态, 也缺乏建立种间关系的研究, 特别是缺乏回归植物 与“神秘”的生物群(真菌、根瘤菌、土壤原生动物等) 间建立连接关系的研究工作, 更缺少授粉、种子扩 散等关键生态系统过程的重建工作。因此, 今后植 物园要在生态系统尺度上关注回归过程中的非生 物障碍和生物障碍等定居限制因子的解除策略, 增 加回归的成功率。

植物园要实现生物多样性的有效保护, 需要综 合考虑就地保护、迁地保护和回归三位一体的综合 保育体系。这其中理由之一就是植物园迁地保育的 物种遗传多样性可能偏小, 且存在近交衰退和杂交
问题, 植物园迁地保育的植物作野外回归要慎重。 在稀有种群回归过程中要考虑遗传多样性, 因为遗 传多样性考虑了进化过程及未来适应不同环境的 问题。多种源的种苗会携带更多的遗传多样性并在 回归过程中有更多的成功机会。此外, 在回归时, 植物园可集成生物技术(组织培养)和生态工程技术, 把材料繁殖 + 生境恢复 + 园艺措施 + 种间关系 恢复进行技术集成, 开展回归种群的生态适应性管 理, 以提高回归成功率, 并最终实现植物多样性有 效保护。目前, 植物园界大多认为植物回归的材料 不能用转基因和基因修饰技术改良。

全球气候变化会导致植物生境的改变, 这对植 物园的迁地保护提出了更高的要求, 同时也为植物 园提供了进行全球变化与生物多样性保护相关联 的研究机会。植物园可以利用收集的活植物预测全 球变化的影响, 也可以开展全球气候变化背景下的植 物的保护性收集(conservation collections, 即以物种 保护为目的, 按照居群生态学和遗传多样性原则从 自然居群取样, 涵盖物种的大部分遗传资源)和回 归。植物回归必须面对当前的全球变化与人类干扰, 要将这些因素纳入回归工作的范畴, 特别是要考虑 “人类世”中人类社会的经济、文化、乡土知识对回 归的正面促进作用或负面影响。还可将回归等保护 工作与生态补偿、自然资本或生态系统服务功能评 估、绿色发展、国际生物多样性保护公约履约相结 合, 在实现生物多样性保护的基础上，保证人类的 福祉并实现可持续发展。

\section{参考文献}

Akeroyd J, Wyse Jackson P (1995) A Handbook for Botanic Gardens on the Reintroduction of Plants to the Wild. BGCI, Richmond, London.

Andel J, Aronson J (2012) Restoration Ecology: The New Frontier, 2nd edn. Blackwell, Chichester.

Armstrong DP, Seddon PJ (2008) Directions in reintroduction biology. Trends in Ecology \& Evolution, 23, 20-25.

Council of Europe (1985) Recommendation No. R (85) 15 of the Committee of Ministers on the Reintroduction of Wildlife Species. Committee of Ministers, Council of Europe, the 388th Meeting of the Ministers' Deputies, Brussels.

Falk DA, Millar CI, Olwell P (1996) Restoring Diversity: Strategies for Reintroduction of Endangered Plants. Island Press, Washington, DC.

Godefroid S, Vanderborght T (2011) Plant reintroductions: the need for a global database. Biodiversity Conservation, 20, 
3683-3688.

Godefroid S, Piazza C, Rossi G, Buord S, Stevens AD, Aguraiuja $\mathrm{R}$, Cowell C, Weekley CW, Vogg G, Iriondo JM, Johnson I, Dixon B, Gordon D, Magnanon S, Valentin B, Bjureke K, Koopman R, Vicens M, Virevaire M, Vanderborght $\mathrm{T}$ (2011) How successful are plant species reintroductions? Biological Conservation, 144, 672-682.

Guerrant EO, Kaye TN (2007) Reintroduction of rare and endangered plants: common factors, questions and approaches. Australian Journal of Botany, 55, 362-370.

He SA, Zhang ZS, Gu Y (2005) Botanical Garden Sciences. China Agriculture Press, Beijing. (in Chinese) [贺善安, 张 佐双, 顾姻 (2005) 植物园学. 中国农业出版社, 北京.]

Heywood VH (2011) The role of botanic gardens as resource and introduction centres in the face of global change. Biodiversity Conservation, 31, 221-239.

Huang HW, Zhang Z (2012) Current status and prospects of ex situ cultivation and conservation of plants in China. Biodiversity Science, 20, 559-571. (in Chinese with English abstract) [黄宏文, 张征 (2012) 中国植物引种栽培及迁地 保护的现状与展望. 生物多样性, 20, 559-571.]

IUCN (1998) Guidelines for Re-introductions. International Union for Conservation of Nature, Gland, Switzerland.

IUCN (2009) Guidelines for the in situ Re-introduction and Translocation of African and Asian Rhinoceros. International Union for Conservation of Nature, Gland, Switzerland.

Jacobs DF, Dalgleish HJ, Nelson CD (2013) A conceptual framework for restoration of threatened plants: the effective model of American chestnut (Castanea dentata) reintroduction. New Phytologist, 197, 378-393.

Liu H, Ren H, Liu Q (2015) The conservation translocation of threatened plants as a conservation measure in China: a review. Conservation Biology, 29, 1537-1551.

Maschinski J, Haskins KE (2012) Plant Reintroduction in a Changing Climate: Promises and Perils. Island Press, Washington, DC.

Maunder M (1992) Plant reintroduction: an overview. Biodiversity and Conservation, 1, 51-61.

Raven PH (2006) Research in botanic gardens. Public Garden, 21, 16-17.

Ren H (2006) Theory and Practice on Construction of Classical Botanical Garden. Science Press, Beijing. (in Chinese) [任 海 (2006) 科学植物园建设的理论与实践. 科学出版社, 北京.]

Ren H, Duan ZY (2017) Theory and Practice on Construction of Classical Botanical Garden, 2nd edn. Science Press, Beijing. (in Chinese) [任海, 段子渊 (2017) 科学植物园建设
的理论与实践(第二版). 科学出版社, 北京.]

Ren H, Ma GH, Zhang QM (2010) Moss is a key nurse plant for reintroduction of the endangered herb, Primulina tabacum Hance. Plant Ecology, 209, 313-320.

Ren H, Zhang QM, Lu HF (2012) Wild plant species with extremely small populations require conservation and reintroduction in China. Ambio, 41, 913-917.

Ren H, Jian SG, Liu HX (2014) Advances in the reintroduction of rare and endangered wild plant species. Science China: Life Sciences, 57, 603-609.

Sainz-Ollero H, Hernandez-Bermejo E (1979) Experimental reintroductions of endangered plant species in their natural habitats in Spain. Biological Conservation, 16, 195-206.

Sharrock S (2012) Global Strategy for Plant Conservation: A Guide to the GSPC, All the Targets, Objectives and Facts. BGCI, London.

Sheean VA, Manning AD, Lindenmayer DB (2012) An assessment of scientific approaches towards species relocations in Australia. Australia Ecology, 37, 204-215.

Sun WB (2013) Conservation of Wild Plants with Extremely Small Population in Yunnan: Practice and Exploration. Yunnan Science and Technology Press, Kunming. (in Chinese) [孙卫邦 (2013) 云南省极小种群野生植物保护: 实 践与探索. 云南科技出版社, 昆明.]

Wang ZF, Ren H, Li ZH, Zhang QM (2013) Local genetic structure in the critically endangered, cave-associated perennial herb Primulina tabacum (Gesneriaceae). Biological Journal of the Linnean Society, 109, 747-756.

Wyse Jackson P, Kennedy K (2009) The global strategy for plant conservation: a challenge and opportunity for the international community. Trends in Plant Science, 14, 578-580.

Wyse Jackson P, Sutherland LA (2012) International Agenda for Botanic Gardens in Conservation. Botanic Gardens Conservation International, Richmond.

Wyse Jackson P, Sutherland LA (2013) Role of Botanic Gardens. In: Encyclopedia of Biodiversity, 2nd edn. Vol. 6 (ed. Levin SA), pp. 504-521. Academic Press, Waltham, MA.

Xu ZF (2005) Study on functional orientation of botanical garden. The Botanical Gardens of China, (8), 1-6. (in Chinese with English abstract) [许再富 (2005) 植物园的功能定位 研究. 中国植物园, (8), 1-6.]

Yam TW, Chua J, Tay F, Ang P (2010) Conservation of the native orchids through seedling culture and reintroduction-a Singapore experience. The Botanical Review, 76, 263-274.

(责任编委: 龙春林 责任编辑: 问文杰) 